\title{
Chronic ulcerative stomatitis: case series of an under-recognized entity
}

(Running title: Chronic ulcerative stomatitis)

Eugene M. Ko, ${ }^{1,2}$ Theodora E. Danciu, ${ }^{1}$ Douglas R. Fullen, ${ }^{3,4}$ May P. Chan ${ }^{3,4}$

${ }^{1}$ Department of Periodontics and Oral Medicine, School of Dentistry, University of Michigan, Ann Arbor, MI, USA

${ }^{2}$ Department of Oral Medicine, School of Dental Medicine, University of Pennsylvania, Philadelphia, PA, USA

${ }^{3}$ Department of Pathology, University of Michigan, Ann Arbor, MI, USA

${ }^{4}$ Department of Dermatology, University of Michigan, Ann Arbor, MI, USA

Corresponding author:

May P. Chan, MD

NCRC Building 35

2800 Plymouth Road

Ann Arbor, MI 48109

Tel: (734)764-4460

Fax: (734)764-4690

Email:mpchan@med.umich.edu

Keywords: Chronic ulcerative stomatitis, lichen planus, antinuclear antibody, direct immunofluorescence

\begin{abstract}
This is the author manuscript accepted for publication and has undergone full peer review but has not been through the copyediting, typesetting, pagination and proofreading process, which may lead to differences between this version and the Version of Record. Please cite this article as doi: 10.1111/cup.13347
\end{abstract}

This article is protected by copyright. All rights reserved. 
Chronic ulcerative stomatitis is a mucocutaneous condition characterized by chronic relapsing and remitting oral ulcers and erosions. This condition remains under-recognized among dermatopathologists, possibly due to common misdiagnosis as oral erosive lichen planus. We report 5 cases of chronic ulcerative stomatitis in order to raise awareness of this uncommon condition. All patients presented with desquamative gingivitis and/or oral erosions, with biopsies showing lichenoid mucositis and epithelial nuclear IgG deposition on direct immunofluorescence. Recognition of the characteristic direct immunofluorescence findings allows for distinction of chronic ulcerative stomatitis from oral lichen planus and appropriate therapy. 


\section{Introduction}

Chronic ulcerative stomatitis (CUS) is characterized clinically by oral erosions or ulcerations with periods of exacerbation and remission. ${ }^{1}$ Since its first description in 1990, a limited number of cases have been reported to date. ${ }^{2}$ Its apparently low incidence is likely due to underrecognition of this condition, as diagnosis requires direct immunofluorescence study in addition to routine light microscopy. We encountered 5 patients who presented with clinical, histopathologic, and direct immunofluorescence findings compatible with CUS. These cases are reported herein to raise awareness of this relatively rare condition among dermatopathologists.

\section{Case Presentations}

\section{Case 1}

A 63-year-old woman presented with painful desquamative gingivitis (Figure 1A). Clinical examination revealed sloughing of the gingival epithelium upon palpation, consistent with a positive Nikolsky sign. Biopsy revealed a band-like lymphocytic infiltrate with apoptosis of occasional basal keratinocytes (Figures 1B and 1C). Direct immunofluorescence examination showed nuclear IgG and IgA (weak) staining within the lower third of the epithelium, as well as shaggy fibrin deposition at a focus of subepithelial split (Figures 1D-1F). 


\section{Case 2}

A 65-year-old woman complained of difficulty wearing her dentures due to tenderness in the posterior mandible. Physical examination revealed redness and leukoplakia of bilateral posterior mandible and maxillary ridges. Clinical impression was oral lichen planus (LP). A biopsy was taken from the left buccal mucosa, which showed lichenoid mucositis with mild epithelial hyperplasia and hyperkeratosis (Figure 2A). The inflammatory infiltrate was composed of predominantly lymphocytes and a few plasma cells. Direct immunofluorescence revealed nuclear binding of $\operatorname{IgG}$ in the basal and suprabasal epithelial keratinocytes (Figure 2B) and shaggy fibrin deposition along the basement membrane zone.

\section{Case 3}

A 61-year-old man with a reported history of oral LP was treated with clobetasol gel and prednisone "swish and spit", which led to improvement but not resolution. Oral examination was remarkable for confluent erythema and erosions of the upper and lower attached gingiva. Skin examination was unremarkable. A punch biopsy was taken from the right gingival buccal sulcus, which demonstrated lichenoid mucositis with basal squamatization and rare cytoid bodies (Figure 3A). Hypergranulosis, hyperkeratosis, and mild squamous atypia were also noted. The lichenoid infiltrate consisted of predominantly lymphocytes and sparse plasma cells. Another biopsy from the anterior buccal mucosa was submitted for direct immunofluorescence study, which revealed nuclear IgG binding of the basal and suprabasal keratinocytes (Figure 3B). 
Serologic testing for antinuclear antibody (ANA) was negative. Hydroxychloroquine treatment was initiated, however, clinical follow-up data was not available.

\section{Case 4}

A 70-year-old woman with a presumed history of erosive LP had biopsies of her oral mucosa in 2012, which reportedly showed lichenoid inflammation and absence of immunoreactants on direct immunofluorescence. These biopsies were not available for review. Despite management with topical steroids, the patient experienced persistent desquamative gingivitis. A second biopsy from the upper gingiva was taken in 2015 which revealed epithelial hyperplasia with patchy lichenoid lymphocytic inflammation and scarring (Figure 4A). A few cytoid bodies were observed in the areas of lichenoid inflammation. Plasma cells were found in the areas of scarring. Direct immunofluorescence showed strong IgG deposition in the epithelial cell nuclei in at least the basal and suprabasal layers; full-thickness evaluation was limited by tangential sectioning. Shaggy fibrin deposition was also noted focally at the basement membrane zone.

\section{Case 5}

An 86-year-old woman presented with generalized atrophic erosive gingiva and hyperkeratotic striae on her oral mucosa. A biopsy of her gingiva was performed in 2007 which revealed lichenoid mucositis. The patient was lost to follow-up until 2017, when another biopsy was taken from the lower right gingiva due to persistent symptoms. Hematoxylin-eosin stain showed variably hyperplastic and atrophic areas with lichenoid inflammation and foci of subepithelial clefting (Figure 4B). A few cytoid bodies were found at and near the cleft. The inflammatory 
infiltrate consisted of both lymphocytes and plasma cells. Direct immunofluorescence revealed IgG deposition in the nuclei of epithelial cells in the basal one-third of the epithelium.

The clinical, histopathologic, and direct immunofluorescence findings in all 5 cases are summarized in Table 1.

\section{Discussion}

All 5 cases in this series displayed characteristic clinical, histopathologic, and immunofluorescence findings of CUS. Clinically, 4 of our patients were middle-aged women, concordant with the strong female predilection and peak of onset in the $5^{\text {th }}$ and $6^{\text {th }}$ decades previously reported in the literature. ${ }^{1-4}$ Almost all areas of the oral cavity can be involved, but the tongue, buccal mucosa, and gingiva are the most commonly affected. ${ }^{3,4}$ Synchronous skin involvement has been rarely reported ${ }^{3}$ but was not observed in our patients. In addition to oral erosions and ulcerations, mucosal erythema and sometimes white striae may be present. When the gingiva is involved, minor manipulation of tissue may cause epithelial sloughing, resulting in desquamative gingivitis. ${ }^{3}$ The latter is a nonspecific sign shared by various mucocutaneous diseases including erosive LP, mucous membrane pemphigoid, pemphigus vulgaris, among others. ${ }^{2,3,5}$ Biopsy is therefore required for definitive diagnosis.

The histopathologic features of CUS most closely resemble those of oral LP in that there is a lichenoid infiltrate consisting of predominantly lymphocytes. With time this results in atrophy 
and ulceration of the squamous epithelium. ${ }^{2}$ There may be subepithelial clefting in the areas of robust inflammation. Scarring may also occur in relapsing cases, as seen in Case 4. Increased number of plasma cells is often associated with ulceration, subepithelial clefting, or scarring. None of our cases contains eosinophils. Parakeratosis is often present. As CUS and oral LP are essentially indistinguishable clinically and histopathologically, ${ }^{2,4}$ diagnosis of CUS relies primarily on direct immunofluorescence study.

On direct immunofluorescence, CUS is characterized by a speckled pattern of IgG deposition in the nuclei of keratinocytes limited to the lower layers of the oral squamous epithelium. ${ }^{3}$ This pattern is due to binding of stratified epithelium-specific antinuclear antibodies (SES-ANAs). Occasionally, IgA and C3 deposition may also be observed in a similar pattern. ${ }^{6}$ The presence of SES-ANA is the diagnostic hallmark of CUS which distinguishes it from oral LP. Other features that may be observed in CUS include shaggy fibrin deposition and cytoid bodies at the basement membrane zone, both of which are nonspecific findings that can also be seen in oral LP and other lichenoid mucositides. ${ }^{4,7}$ Importantly, biopsy for direct immunofluorescence should be taken from perilesional intact mucosa, as ulcerated areas are devoid of epithelium and would yield non-diagnostic results.

Indirect immunofluorescence may be used as an adjunctive test to support a diagnosis of CUS, especially if the biopsy specimen is difficult to interpret due to poor tissue orientation. ${ }^{1,3,8,9}$ Circulating IgG SES-ANAs can be demonstrated in the sera of patients with CUS using monkey 
or guinea pig esophagus (squamous epithelium) as tissue substrate. ${ }^{6}$ The same speckled nuclear staining pattern as described on direct immunofluorescence is expected.

The antigen targeted by SES-ANA was initially found to be a $70 \mathrm{kDa}$ protein. ${ }^{10}$ Lee et al. identified this protein to be an isoform of p63 ("Np63 ), referred to as the chronic ulcerative stomatitis protein (CUSP). ${ }^{11}$ This has led to the development of an enzyme-linked immunosorbent assay (ELISA) using the N-terminal portion of " Np63 \pm to detect the presence of CUSP in patients' sera. ${ }^{12}$ Expression of p63 is critical for the development of stratified squamous epithelia. ${ }^{13-15}$ Specifically, "Np63 \pm may have a role in maintaining the viability and proliferative capacity of the basal epithelial cells, as well as blocking the consequences of inappropriate p53 activation. ${ }^{13,16}$ Therefore, by interfering with the presumed functions of "Np63 \pm , SES-ANA may cause epithelial breakdown and detachment, leading to oral erosions and ulcerations as seen in CUS. $^{12}$

The major differential diagnosis of CUS includes oral LP and connective tissue disease. As mentioned above, the clinical and histopathologic features of CUS and oral LP are essentially identical. Indeed, 2 of our patients carried a past diagnosis of oral LP. While epithelial nuclear IgG binding in CUS serves as a defining discriminator, autoantibodies directed to a $70 \mathrm{kDa}$ antigen (presumably SES-ANAs) have also been detected in the sera of some patients with LP. ${ }^{17-}$

${ }^{19}$ Parodi et al. tested the sera of 138 patients with skin or mucosal LP using monkey esophagus and HEp2-2000 cells as substrates, and found SES-ANAs directed against a $70 \mathrm{kDa}$ antigen in 9 (7\%) patients. ${ }^{17}$ These findings have led some to regard CUS as a variant of LP. ${ }^{18}$ However, the 
identity of this $70 \mathrm{kDa}$ antigen is not entirely clear as it was not sequenced or detected with a control monoclonal antibody to $" \mathrm{~Np} 63 \pm$ in the aforementioned study. ${ }^{12}$ Furthermore, as direct immunofluorescence was not performed, one could speculate whether some of their cases may indeed be CUS misclassified as LP. Clinically, unlike oral LP which typically responds to corticosteroids, CUS is usually refractory to corticosteroids but improves dramatically with hydroxychloroquine, thus providing additional evidence that these 2 conditions should be regarded as distinct pathologic entities. ${ }^{2,3,6,12}$

A similar nuclear binding pattern may be seen in patients with circulating ANAs, a finding that is often associated with connective tissue diseases. Binding of ANAs to keratinocytes in connective tissue diseases typically involves the entire thickness of the epithelium, ${ }^{6,20}$ whereas binding of SES-ANAs in CUS is usually confined to the lower one-third of the epithelium. Assessment for this distribution pattern requires proper orientation of the mucosal tissue, as tangential sectioning may result in false impression of full-thickness deposition. ${ }^{1}$ From our experience, the intensity of SES-ANA in CUS tends to be stronger than ANA in connective tissue diseases. Ultimately, correlation with ANA serologies should be considered in patients with clinical stigmata of connective tissue diseases. None of our patients had a known history of connective tissue disease or known positive ANA serologies, although the latter was performed in one patient only, and the available clinical information was rather limited in all patients. This remains a potential confounding factor and limitation of our series. 
In conclusion, CUS can be easily misdiagnosed in the absence of direct immunofluorescence examination. As such, direct immunofluorescence study would be helpful in determining the most appropriate therapy when a lichenoid mucositis is first diagnosed, or when a presumed case of oral LP fails to respond to corticosteroids. Once nuclear IgG binding is demonstrated, correlation with any clinical and/or serologic evidence of connective tissue disease is recommended before a diagnosis of CUS is confirmed.

\section{References}

1. Solomon LW, Aguirre A, Neiders M, et al. Chronic ulcerative stomatitis: clinical, histopathologic, and immunopathologic findings. Oral Surg Oral Med Oral Pathol Oral Radiol Endod. 2003;96(6):718-726.

2. Cheng YS, Gould A, Kurago Z, Fantasia J, Muller S. Diagnosis of oral lichen planus: a position paper of the American Academy of Oral and Maxillofacial Pathology. Oral Surg Oral Med Oral Pathol Oral Radiol. 2016;122(3):332-354.

3. Islam MN, Cohen DM, Ojha J, Stewart CM, Katz J, Bhattacharyya I. Chronic ulcerative stomatitis: Diagnostic and management challenges-four new cases and review of literature. Oral Surg Oral Med Oral Pathol Oral Radiol Endod. 2007;104(2):194-203.

4. Qari H, Villasante C, Richert J, Rees T, Kessler H. The diagnostic challenges of separating chronic ulcerative stomatitis from oral lichen planus. Oral Surg Oral Med Oral Pathol Oral Radiol. 2015;120(5):622-627. 
5. Scully C, Porter SR. The clinical spectrum of desquamative gingivitis. Semin Cutan Med Surg. 1997;16(4):308-313.

6. Solomon L. Chronic ulcerative stomatitis. Oral Dis. 2008;14(5):383-389.

7. Kulthanan K, Jiamton S, Varothai S, Pinkaew S, Sutthipinittharm P. Direct immunofluorescence study in patients with lichen planus. Int J Dermatol. 2007;46(12):1237-1241.

8. Alshagroud R, Neiders M, Kramer JM, Suresh L. Clinicopathologic significance of in vivo antinuclear autoantibodies in oral mucosal biopsies. Oral Surg Oral Med Oral Pathol Oral Radiol. 2017;124(5):475-482.

9. Solomon LW, Neiders ME, Zwick MG, Kirkwood KL, Kumar V. Autoimmunity to deltaNp63alpha in chronic ulcerative stomatitis. J Dent Res. 2007;86(9):826-831.

10. Parodi A, Cozzani E, Chorzelski TP, Beutner EH, Rebora A. A molecule of about $70 \mathrm{kd}$ is the immunologic marker of chronic ulcerative stomatitis. J Am Acad Dermatol. 1998;38(6 Pt 1):10051006.

11. Lee LA, Walsh P, Prater CA, et al. Characterization of an autoantigen associated with chronic ulcerative stomatitis: the CUSP autoantigen is a member of the p53 family. J Invest Dermatol. 1999;113(2):146-151.

12. Solomon LW, Stark PC, Winter L, Kumar V, Sinha S. ELISA test for p63 antibodies in chronic ulcerative stomatitis. Oral Dis. 2010;16(2):151-155.

13. Barbieri CE, Pietenpol JA. p63 and epithelial biology. Exp Cell Res. 2006;312(6):695-706.

14. Dellavalle RP, Egbert TB, Marchbank A, Su LJ, Lee LA, Walsh P. CUSP/p63 expression in rat and human tissues. J Dermatol Sci. 2001;27(2):82-87. 
15. Yang A, Schweitzer R, Sun D, et al. p63 is essential for regenerative proliferation in limb, craniofacial and epithelial development. Nature. 1999;398(6729):714-718.

16. Marchbank A, Su LJ, Walsh P, et al. The CUSP DeltaNp63alpha isoform of human p63 is downregulated by solar-simulated ultraviolet radiation. J Dermatol Sci. 2003;32(1):71-74.

17. Parodi A, Cozzani E, Massone C, et al. Prevalence of stratified epithelium-specific antinuclear antibodies in 138 patients with lichen planus. J Am Acad Dermatol. 2007;56(6):974-978.

18. Feller L, Khammissa RAG, Lemmer J. Is chronic ulcerative stomatitis a variant of lichen planus, or a distinct disease? J Oral Pathol Med. 2017;46(10):859-863.

19. Cozzani E, Cacciapuoti M, di Marco E, Zerega B, Descalzi Cancedda F, Parodi A. Patients with oral erosive and cutaneous lichen planus may have antibodies directed against the chronic ulcerative stomatitis protein antigen of 70-kDa. Acta Dermatovenerol Alp Pannonica Adriat. 2008;17(3):120-124.

20. Koelle MS, Kumar V, Beutner EH, Chorzelski TP. In-vivo epidermal nuclear reactions: a selective process. Br J Dermatol. 1991;125(1):48-52.

This article is protected by copyright. All rights reserved. 


\section{Figure Legends}

Figure 1. Case 1. A) The patient presented with desquamative gingivitis most prominent in the upper gingiva. B) Biopsy showed lichenoid mucositis. C) The infiltrate is composed of predominantly lymphocytes associated with interface changes including occasional cytoid bodies. D) Direct immunofluorescence revealed strong nuclear IgG staining within the lower 
third of the epithelium. E) A similar staining pattern was seen with IgA. F) A focus of subepithelial split also demonstrated shaggy fibrin deposition. (B and C, hematoxylin-eosin stain; D, IgG immunofluorescence; E, IgA immunofluorescence; F, fibrin immunofluorescence; original magnifications $\times 100[\mathrm{~B}]$ and $\times 200[\mathrm{C}-\mathrm{F}])$

Figure 2. Case 2. A) Biopsy of the buccal mucosa showed a band-like lymphocytic infiltrate composed of predominantly lymphocytes. Hyperkeratosis and mild reactive squamous atypia were also noted. B) Direct immunofluorescence revealed speckled nuclear deposits of IgG in the basal and suprabasal epithelial keratinocytes. (A, hematoxylin-eosin stain, original magnifications $\times 200$ and $\times 40$ (inset); $\mathrm{B}, \operatorname{IgG}$ immunofluorescence, original magnification $\times 200$ )

Figure 3. Case 3. A) Biopsy of the gingival buccal sulcus demonstrated lichenoid mucositis with a few cytoid bodies. Hypergranulosis, hyperkeratosis, and mild reactive squamous atypia were also noted. B) Direct immunofluorescence of another biopsy from the buccal mucosa revealed nuclear IgG binding of the basal and suprabasal keratinocytes. (A, hematoxylin-eosin stain, original magnifications $\times 200$ and $\times 40$ (inset); $\mathrm{B}, \operatorname{IgG}$ immunofluorescence, original magnification $\times 200$ )

Figure 4. Cases 4 and 5. A) Biopsy from the upper gingiva of patient 4 revealed epithelial hyperplasia with patchy lichenoid inflammation (right) and scarring (left). Cytoid bodies were present in the lichenoid areas (inset). B) Biopsy from the lower gingiva of patient 5 was remarkable for lichenoid mucositis with partial subepithelial split. Cytoid bodies were seen at 
and near the split. (Hematoxylin-eosin stain, original magnifications $\times 40[\mathrm{~A}], \times 200[\mathrm{~A}$, inset], and $\times 200[\mathrm{~B}])$ 

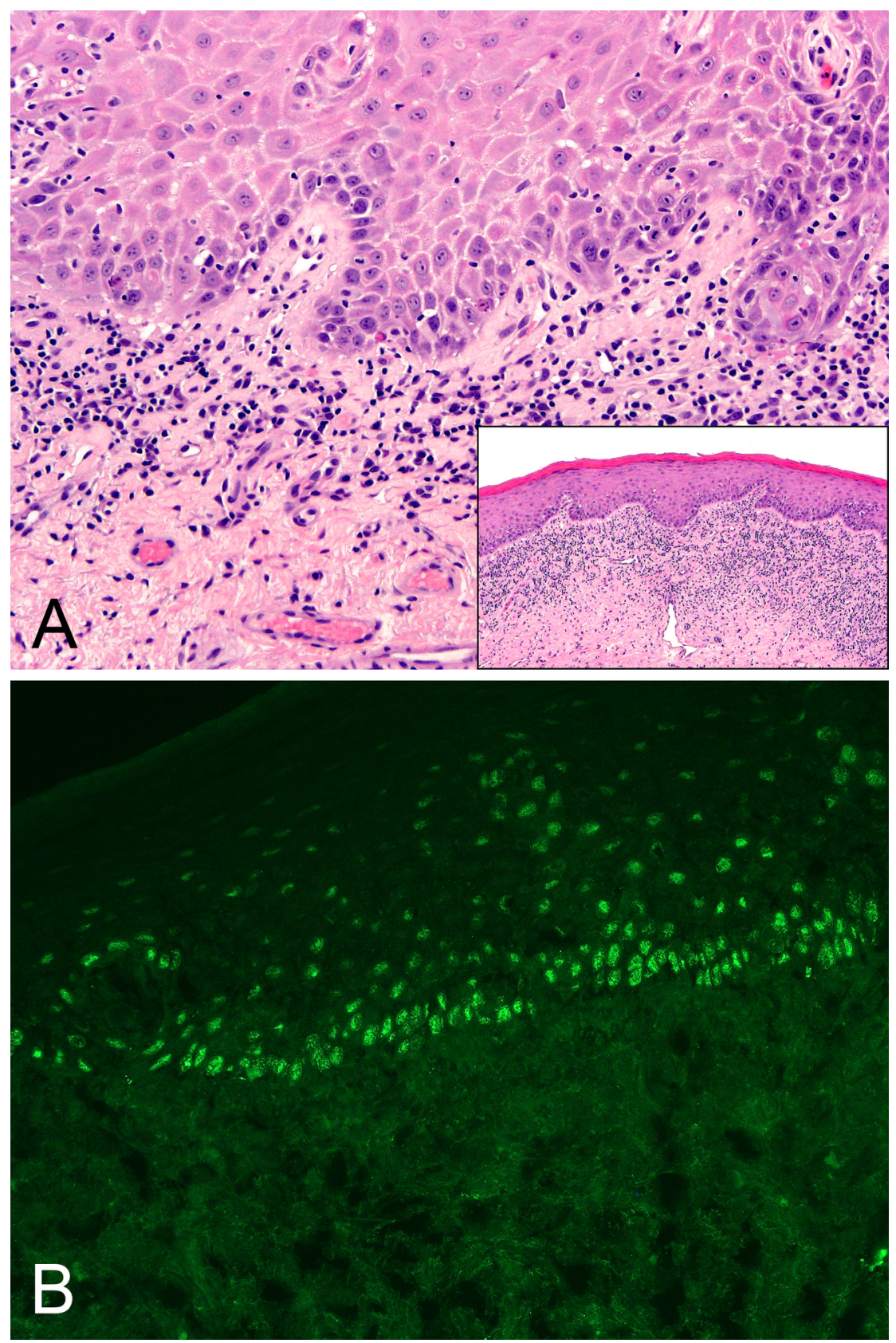

Fig2.tif

This article is protected by copyright. All rights reserved. 


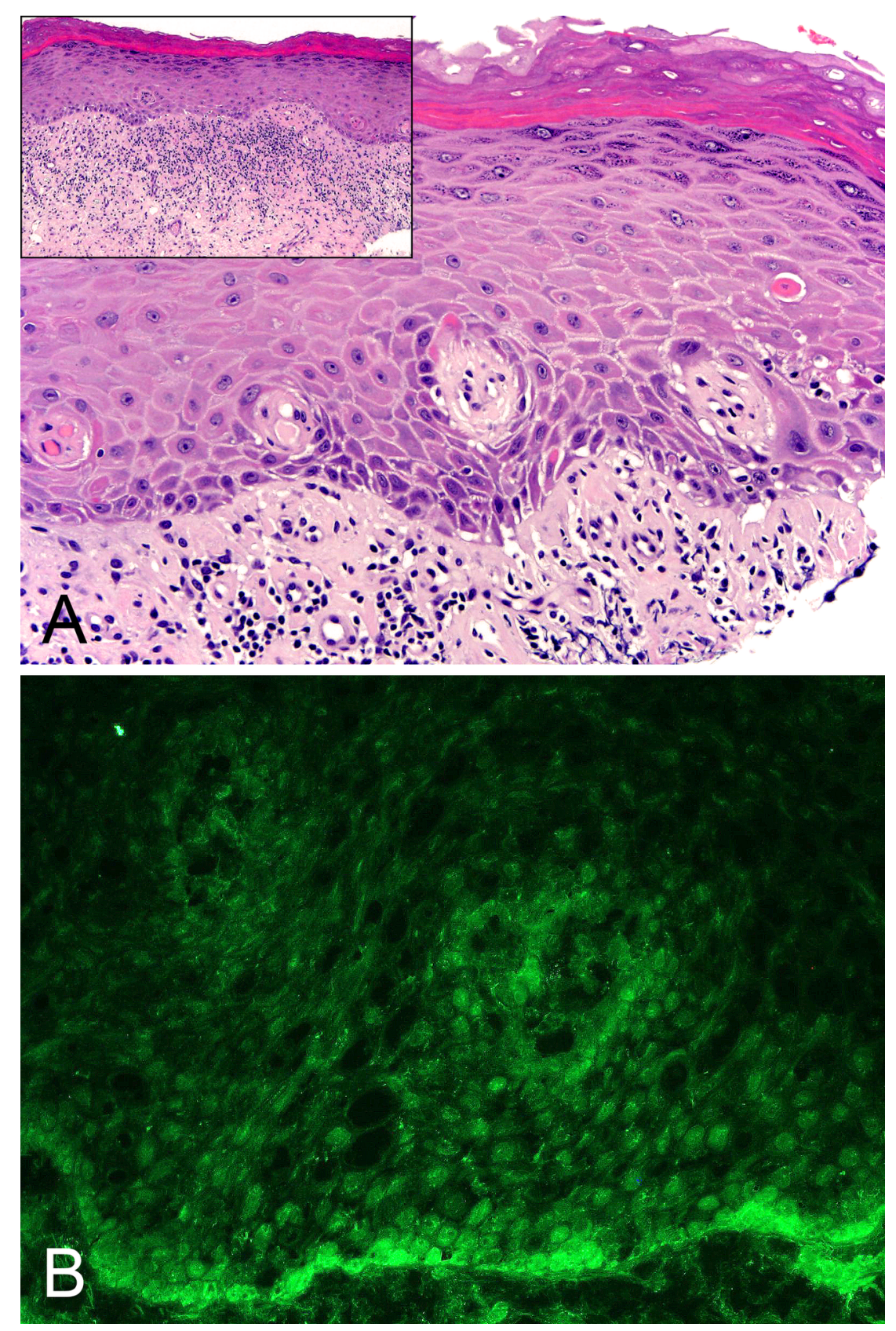

Fig3.tif

This article is protected by copyright. All rights reserved. 

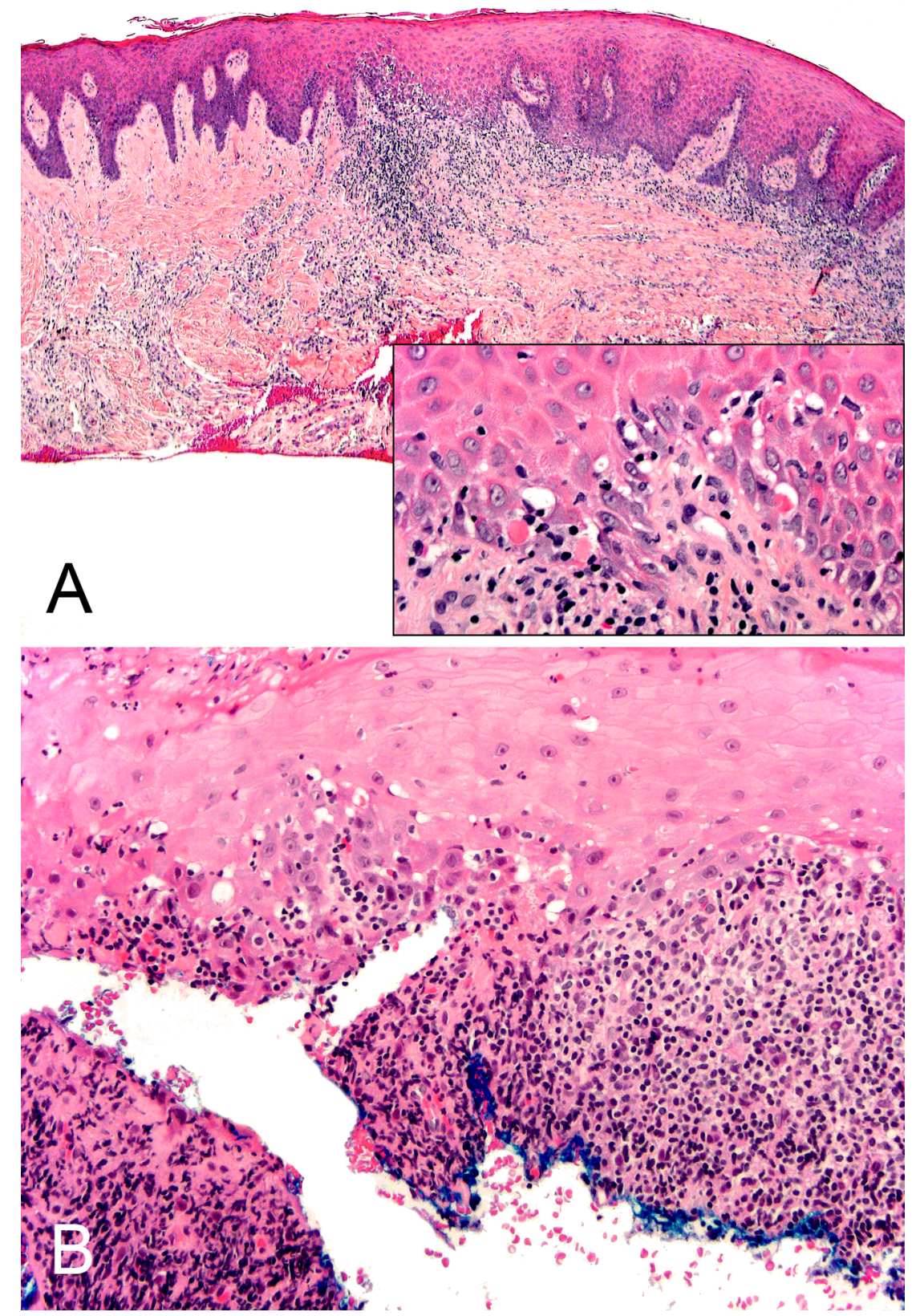

Fig4.tif

This article is protected by copyright. All rights reserved. 
Table 1. Summary of clinical, histopathologic, and direct immunofluorescence findings.

\begin{tabular}{|c|c|c|c|c|c|}
\hline & Case 1 & Case 2 & Case 3 & Case 4 & Case 5 \\
\hline \multicolumn{6}{|l|}{ Clinical } \\
\hline Age/sex & $63 / F$ & $65 / F$ & $61 / \mathrm{M}$ & $70 / F$ & $86 / F$ \\
\hline Positive ANA serologies & NK & NK & - & NK & NK \\
\hline Oral ulcer/erosion & - & - & + & + & + \\
\hline Desquamative gingivitis & + & - & + & + & + \\
\hline $\begin{array}{l}\text { Resolution with topical } \\
\text { steroid }\end{array}$ & NK & NK & - & - & NK \\
\hline Relapsing course & NK & NK & + & + & + \\
\hline \multicolumn{6}{|l|}{ Histopathology } \\
\hline $\begin{array}{l}\text { Lichenoid lymphocytic } \\
\text { inflammation }\end{array}$ & + & + & + & + & + \\
\hline Plasma cells & - & + & + & + & + \\
\hline Dyskeratoses/cytoid bodies & + & + & + & + & + \\
\hline Epithelial hyperplasia & - & + & + & + & + \\
\hline Hyperkeratosis & - & + & + & - & + \\
\hline \multicolumn{6}{|l|}{ Direct Immunofluorescence } \\
\hline Nuclear IgG* & + & + & + & + & + \\
\hline Nuclear IgA* & + (weak) & - & - & - & - \\
\hline Shaggy fibrin at BMZ & + & - & - & + & - \\
\hline
\end{tabular}

*Nuclear immune deposition confined to lower epithelium

ANA, antinuclear antibody; BMZ, basement membrane zone; $F$, female; $M$, male; NK, not known or not applicable; +, present; -, absent. 


\section{Chronic ulcerative stomatitis: case series of an under-recognized entity}

(Running title: Chronic ulcerative stomatitis)

Eugene M. Ko, ${ }^{1,2}$ Theodora E. Danciu, ${ }^{1}$ Douglas R. Fullen, ${ }^{3,4}$ May P. Chan ${ }^{3,4}$

${ }^{1}$ Department of Periodontics and Oral Medicine, School of Dentistry, University of Michigan,

Ann Arbor, MI, USA

${ }^{2}$ Department of Oral Medicine, School of Dental Medicine, University of Pennsylvania,

Philadelphia, PA, USA

${ }^{3}$ Department of Pathology, University of Michigan, Ann Arbor, MI, USA

${ }^{4}$ Department of Dermatology, University of Michigan, Ann Arbor, MI, USA

Corresponding author:

May P. Chan, MD

NCRC Building 35

2800 Plymouth Road

Ann Arbor, MI 48109

Tel: (734)764-4460

Fax: (734)764-4690

Email:mpchan@med.umich.edu

Keywords: Chronic ulcerative stomatitis, lichen planus, antinuclear antibody, direct immunofluorescence 